\title{
UNDERWORLDS: Cascading Situation Assessment for Robots
}

\author{
Séverin Lemaignan ${ }^{1}$, Yoan Sallami ${ }^{2}$, Christopher Wallbridge ${ }^{3}$, \\ Aurélie Clodic ${ }^{2}$, Tony Belpaeme ${ }^{3}$, and Rachid Alami ${ }^{2}$
}

\begin{abstract}
We introduce UNDERWORLDS, a novel lightweight framework for cascading spatio-temporal situation assessment in robotics. UNDERWORLDS allows programmers to represent the robot's environment as real-time distributed data structures, containing both scene graphs (for representation of 3D geometries) and timelines (for representation of temporal events). UNDERWORLDS supports cascading representations: the environment is viewed as a set of worlds that can each have different spatial and temporal granularities, and may inherit from each other. UNDERWORLDS also provides a set of highlevel client libraries and tools to introspect and manipulate the environment models.
\end{abstract}

This article presents the design and architecture of this open-source tool, and explores some applications, along with examples of use.

\section{INTRODUCTION}

UNDERWORLDS is a distributed and lightweight opensource framework 1 that enables robot programmers to build and refine spatial and temporal models of the environment surrounding a robot in real-time. UNDERWORLDS makes it possible to share these world models amongst the software components running on the robot. Additionally, UNDERWORLDS enables users to represent and manipulate multiple alternatives to the current, perceived world model in a distributed manner. For instance, the world with some objects filtered out; the world 'viewed' from the perspective of another agent; a hypothetical world resulting from the simulated application of a plan, etc.

\section{A. Distributed Situation Assessment}

Anchoring perceptions in a symbolic model suitable for decision-making requires perception abilities and their symbolic interpretation. We call physical situation assessment the cognitive skill that a robot exhibits when it represents and assesses the nature and content of its surroundings and monitors its evolution.

Numerous approaches exist, like amodal (in the sense of modality-independent) proxies [1], grounded amodal representations [2], semantic maps [3], [4], [5] or affordancebased planning and object classification [6], [7].

UNDERWORLDS is specifically inspired by geometric and temporal reasoners like SPARK (SPAtial Reasoning \& Knowledge) [8] or TOASTER (Tracking Of Agents and Spatio-

\footnotetext{
${ }^{1}$ Author is with Bristol Robotics Lab, University of the West of England, Bristol, United Kingdom severin.lemaignanebrl.ac.uk, ${ }^{2}$ Authors are with LAAS-CNRS, Université de Toulouse, CNRS, Toulouse, France firstname.surnamedlaas.fr, ${ }^{3}$ Authors are with CRNS, Plymouth University, Plymouth, United Kingdom firstname.surnamedplymouth.ac.uk

1 https://github.com/underworlds-robot/underworlds
}

TEmporal Reasoning) [9]. SPARK acts as a situation assessment reasoner that generates symbolic knowledge from the geometry of the environment with respect to relations between objects, robots and humans. It also takes into account the different perspective that each agent has on the environment. SPARK embeds a modality-independent geometric model of the environment that serves both as basis for the fusion of the perception modalities and as bridge with the symbolic layer [10]. This geometric model is built from 3D CAD models of the objects, furniture and robots, and full body, rigged models of humans. It is updated at run-time by the robot's sensors. Likewise, UNDERWORLDS embeds a grounded amodal model of the environment, updated online from the robot's sensors (sensor fusion).

However, SPARK is a monolithic module that does not support sharing its internal 3D model with other external components. In contrast, UNDERWORLDS focuses on offering a shared and distributed representation of the environment within the robot's software architecture. This also distinguishes UNDERWORLDS from complex cognitive toolkits like KnowRob (as found in OpenEASE [11]). While these tools maintain a spatio-temporal model of the world, this model is internal and not meant to be made widely accessible to other external processes. UNDERWORLDS focuses instead on reusability and sharing of distributed spatio-temporal models. As such, UNDERWORLDS can be seen as a middleware for spatio-temporal world models and, contrary to KnowRob, it does not provide any intrinsic high-level processing or reasoning capability. Such reasoning skills are implemented in loosely-coupled clients (see Section III hereafter).

Work on distributed scene graphs [12] has been previously applied to robotics to provide a shared 3D representation of the robot's environment (for instance, the Robot Scene Graph [13] or the Deep State Representation proposed in [14]). UNDERWORLDS offers a similar distribution mechanism for 3D scene graphs and extends it to temporal representations. Besides, UNDERWORLDS further extends this line of work by providing the ability to create, manipulate and share multiple alternative worlds. As an example, these could correspond to filtered or hypothetical views on the initial, perceived model of the environment.

\section{B. Representing Alternative States of the World}

The components which make use of spatial and temporal models of the environment are usually found in the intermediate layers of robotic architectures, between the lowlevel perceptual layers, and the high-level decisional layers. They include modules like geometric reasoners (that compute 
spatial and topological relations between objects), motion planners or action recognition modules.

These components exhibit different needs in terms of representation, like different nominal spatial and/or temporal resolutions. For instance, a 3D motion planner would typically use coarse 3D models of surrounding objects to lower the computational load while planning, while a module assessing the visibility of objects might need high-resolution models for accurate 3D visibility testing. This requirement of multiple task-specific representations has been framed as the need for deep representations by Beetz [15].

Traditional robotic middlewares, like ROS, are not particularly well suited to deal with these different needs: full geometric data can be represented, but is not firstclass citizen: a basic task like displaying a 3D mesh at an arbitrary position is not particularly easy to perform with ROS, requiring the combination of static Collada meshes, a URDF kinematic description, TF broadcasters, and a 3D visualisation tool like RViz. Critically, simultaneously representing and reasoning on alternative states of the environment is not directly feasible.

Representing alternative states is however often highly desirable. For instance, software components manipulating environment models typically perform better if the models are physically consistent. However, low-level perception inaccuracies often introduce hard-to-avoid physical inconsistencies (like detected objects floating in the air, or wrongly inset into other objects). Therefore, a post-process stage (for instance, using a physics simulation engine) is needed to move the objects seen by the robot into physicallycorrect positions. Implemented with a classical approach (for instance, using ROS TF frames), we would represent an object book with two frames: the original frame (e.g.,book_frame_raw) and a second one computed by the physics engine (e.g.,book_frame_corrected). Such an approach leads to the robot's 3D model being cluttered with multiple frames and does not scale well.

Another example pertains to geometric task planning: a geometric task planner typically needs to reason over hypothetical future states of the environment ("What happens if I move this glass onto that pile of books?"). The planner generates many possible future states, which in turn might require further processing (for instance, running a physics simulation). Such a tool would benefit a flexible representation system, where models are derived from each other, with partial modifications and different timescales.

A third example relates to human-robot interaction scenarios where perspective taking is important (a prototypical example being the game 'I spy with my little eye', as implemented in [16]). Perspective taking is a cognitive skill that relies on the ability for an agent to take someone else's point of view to estimate what they see from their perspectives. Perspective taking has previously been implemented in robotics by temporarily placing virtual cameras at eye locations for each of the humans tracked by the robot [17]. While acceptable for simple cases, such an approach does not maintain truly independent spatio-temporal models of the environment for each agent, and in particular, it does not permit the representation of proper false-belief situations. On the contrary, separate, independent world models as implemented by UNDERWORLDS effectively support such a skill, which is an important precursor to research and implement human's mind modelling (i.e., a theory of mind) [18].

Lastly, geometric pre-supposition accommodation makes another interesting case for alternative worlds representation. Pre-supposition accommodation originally comes from linguistics, where it describes the mechanism by which context is adjusted [...] to accept [...] a sentence that imposes certain requirements on the context in which it is processed [19]. In the context of spatio-temporal representations, we call presupposition accommodation the ability of an agent to adjust its model so that it matches some contextual constraint. For instance, if A tells B to "catch the red balloon behind you", B might create a representation of an imaginary red balloon, placed behind her, even without actually observing the balloon: B accommodates the pre-supposition of a red balloon being present behind herself. Endowing robots with this capability has been touched upon by Mavridis et al. within their multi-modal Grounded Situation Model [2]. However, to the best of our knowledge, a general framework which would enable robots to accommodate spatial and temporal pre-suppositions by deriving imaginary worlds from existing ones has not been proposed so far.

UNDERWORLDS addresses this need and the main contribution of this work is a generic approach to represent and share multiple parallel representations of the world. UNDERWORLDS does so by allowing clients to clone existing worlds, modify them, and re-share them, without the cost of duplicating geometric data (as explained in section III). By organising clients in a network (Figure 1), worlds can be made dependent on each other, resulting in a loosely-coupled modular approach to spatio-temporal world representation that we call cascading situation assessment.

\section{DESIGN AND ARChiteCtURE}

\section{A. Software architecture}

Figure 1 depicts a typical UNDERWORLDS topology: a graph (that happens to be an acyclic graph on Figure 1 but does not have to be in the general case) of worlds, with clients connecting the worlds to each others.

1) Clients: Software components implementing accessing UNDERWORLDS worlds are called clients. Clients can both read and write onto the worlds they are connected to, and automatically see updates broadcast by other clients connected to the same world. To ensure data consistency, worlds can have many simultaneous readers, but only one writer at a given time.

UNDERWORLDS provides several standard clients (like a $3 \mathrm{D}$ visualisation tool or a physics engine simulator). Clients are however typically written by the end users, depending on the needs of one's specific architecture.

2) Worlds: Worlds are effectively distributed data structures composed of a scene graph representing the 3D ge- 


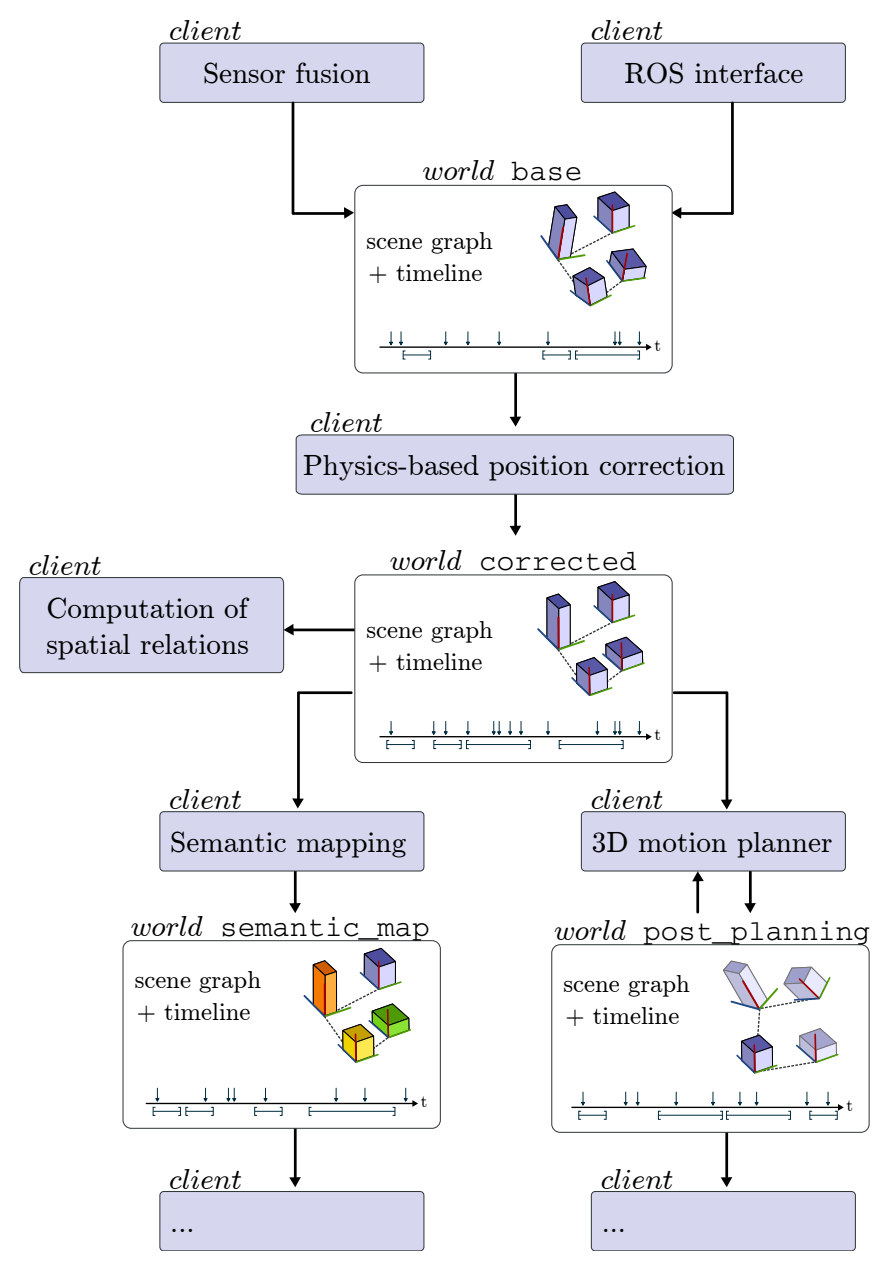

Fig. 1. Schema of a possible UNDERWORLDS network: eight clients (userwritten \& architecture specific; in blue) are sharing environment models through four independent worlds (made from joint spatial and temporal models). This architecture enables successive and modular refinement of the models (cascading situation assessment), effectively adapted to each client's needs.

ometry of the environment, and a timeline storing temporal events.

While each world is technically independent from all the others, dependencies (and therefore, coupling) arise between worlds from the clients' connections. For instance, filters effectively create a dependency between worlds. On Figure 1. the Physics-based position correction client creates a dependency between the world base (which represents here the result of raw sensor fusion) and the world corrected which would be a physically-consistent copy of base. As a result, an UNDERWORLDS network can also be seen as a dependency graph between worlds (where cyclic dependencies are permissible).

This architecture enables what we call cascading situation assessment: independent software components (the clients) build, refine and share successive models of the environment by a combination of filtering/transformations steps and model branching. A change performed by one client (for instance, a face tracker updates the pose of the human head) may thereby cascade to each of the downstream, dependent worlds.

3) Scenes: Worlds contain both a geometric model and a temporal model. The geometric model is represented as a scene graph. The scene graph has a unique root node, to which a tree of other nodes is parented.

Nodes in an UNDERWORLDS scene graph have three possible types: objects that represent concrete physical objects (typically with one or several associated 3D meshes); entities that represent abstract entities like reference frames or groups of objects; perspectives that represent viewpoints of the scene (like cameras or human gaze).

Every node has a unique ID, a parent, a 3D transformation relative to the parent and an optional name. Object nodes optionally store as well pointers to their associated meshes. Importantly, mesh data (or other geometric datasets like point clouds) are not stored within the nodes themselves. UNDERWORLDS represents geometric data as immutable data, identified by their hash value (preventing de facto data duplication). Nodes only store the hash corresponding to the desired geometric data, and the actual data is pulled from the server by the clients whenever they actually need it (for rendering for instance).

4) Timelines: Complementing the spatial representation encapsulated in the scene graph, each world also stores the world's timeline. This data structure is shared and synchronised amongst the clients in the same way as the scene graph. Clients can record and query both events (durationless states) and situations in the timeline, i.e., states with a start time and a (possibly open-ended) end time.

\section{B. Distributed spatio-temporal models}

UNDERWORLDS is not a monolithic piece of software. Instead, it stands for both a network of interconnected clients which manipulate spatial and temporal models of the robot environment (for instance, a motion planner, a object detection module, a human skeleton tracker, etc.), and for a client library that makes it possible to interface existing software components with the network.

Critically, the network is essentially hidden to the client: from the user perspective, the environment model is manipulated as a local data structure (see Listing 1). Modifications to the model are asynchronously synchronised with a central server (the underworlded daemon) and broadcast to every other client connected to the same world.

As previously mentioned, worlds are composite data structures comprised of a scene graph and a timeline. These data structures are synchronised using Google's gRPC message passing framework $2^{2}$ ensuring high throughput, reliability and cross-platform/cross-language support. The UNDERWORLDS API is specifically discussed hereafter, in section

UNDERWORLDS is meant to broadcast complex environment representations (typically including large geometric datasets, like meshes) in real-time. UNDERWORLDS itself does not perform many CPU intensive tasks (CPU intensive

http://www.grpc.io/ 
processing tasks - sensor fusion, physics simulation, etc.are performed by the clients themselves) and as such, the performance bottleneck is essentially the network's data throughput. In that regard, one of the simple yet critical optimisations performed by UNDERWORLDS is automatic caching of mesh data. Mesh data are not transmitted when nodes are updated; only a hash value of the mesh data. The client can then request the full data whenever it is actually needed.

\section{Time and space complexity analysis}

UNDERWORLDS is fundamentally about distributing two datastructures: a scene graph (with nodes representing spatial entities) and a timeline (where events are stored as a flat list). Typical time and space complexities arise from these datastructures. In typical usage scenarios (where the number of nodes or events remain under a few hundred relatively small), the computational load to manipulate these datastructures is however dominated by the actual processings performed by the clients with the data. In the current implementation, scene graphs and timelines are stored in-memory. Were they required, serialization and persistent storage are not anticipated to be difficult to implement.

More interesting is the time complexity of distributing changes across an UNDERWORLDS network. With $n$ the number of worlds and $m$ the number of clients in an UNDERWORLDS network, the worst-case (when every world is a parameter of every client) time complexity of creating or updating a node and propagating the change across the network is $O(n \times m)$ (this effectively corresponds to the UNDERWORLDS server performing $n \times m$ requests to notify clients of the update). The space complexity is the same (as clients own a full copy of the worlds they monitor), except for mesh data whose space and time complexities are $O(1)$ (only the server stores the mesh data).

In the common case of one client performing a full update of a single world (with $p$ nodes) at each time step, the complexity of propagating these changes across the network would be $O(p \times m)$. Figure 2 shows measured propagation time for one change across up to 20 cascading worlds.

\section{API \& CLIENTS}

\section{A. API}

As mentioned, UNDERWORLDS uses Google's gRPC as message passing protocol. The protocol is explicitly defined (using the protocol buffers ${ }^{3}$ interface definition language), and bindings to various languages and platforms can be automatically generated from the protocol definition file (as of Jan 2018, gRPC can generate bindings for C, C++, C\#, Node.js, PHP, Ruby, Python, Go and Java, on Windows, Mac, Linux and Android). The cross-platform/cross-language support of gRPC is especially welcome in the academic context, as it offers ease and flexibility to plug a variety of preexisting components into an UNDERWORLDS network.

https://developers.google.com/protocol-buffers/

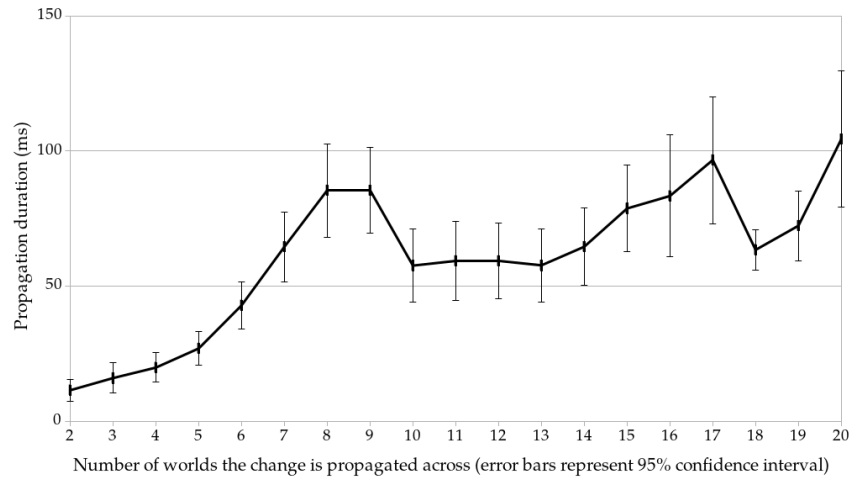

Fig. 2. Propagation times of one change (node creation) across $n$ worlds. The test is performed by running $n-1$ pass-through filters that monitor one world and replicate any changes into the next world. Durations measured over 20 runs, performed on a 8 core machine.

However, the gRPC message passing layer is low-level with respect to the typical use of UNDERWORLDS (manipulation of asynchronous, distributed spatio-temporal models of the robot environment). In particular, the asynchronous fetching (and conversely, remote updating) of nodes and time-related objects is typically hidden from the user, and managed instead by the UNDERWORLDS client library.

UNDERWORLDS currently offers such a high-level client library for Python only (a C++ library is under development). Listing 1 gives a complete example of an UNDERWORLDS client performing simple filtering: the client continuously listens for changes in an input world, removes some objects (in this case, items whose volume is below a threshold), and forwards all other changes to an output world, effectively making the output world a copy of the input world with all smaller objects removed.

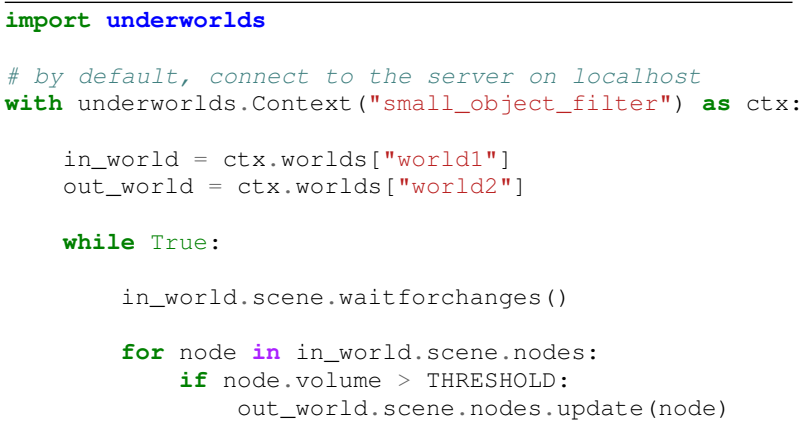

Listing 1: Example of a simple yet complete UNDERWORLDS filter, written in Python: the client connects to the UNDERWORLDS network, blocks until the world world1 changes, and only propagate nodes that match the condition to the world world2.

\section{B. Standard Clients}

The UNDERWORLDS package provides several standard clients to perform common tasks on UNDERWORLDS networks. 


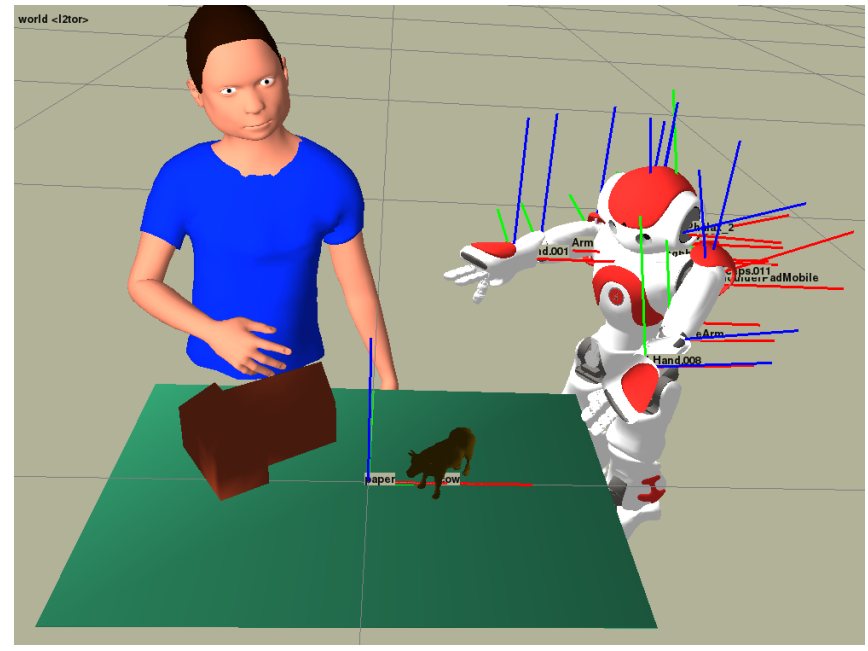

Fig. 3. Screenshot of the uwds view 3D visualisation and manipulation client. In this particular example, the 3D meshes have been pre-loaded using uwds load. Their positions are then updated at run-time using the robot's sensors and proprioception (joint state)

1) $3 D$ Visualisation and manipulation: Interestingly, while UNDERWORLDS deals with 3D geometries and scenes, it does represent 3D entities purely as data structures; no visual representation is involved (and as such, the UNDERWORLDS server and core libraries do not depend on any graphics library like OpenGL). However, for all practical purposes, the ability to visualise the content of a scene is desirable. UNDERWORLDS provides a standard client, uwds view, that performs real-time 3D rendering of worlds, using OpenGL (Figure 3).

This tool also supports basic object manipulations (translations, rotations), that are broadcast to the other UNDERWORLDS clients connected to the same world.

Assets loading: Often, objects manipulated by the robot have known meshes with corresponding CAD models that can be conveniently pre-loaded. In these cases, UNDERWORLDS provides a tool, uwds load, that loads a mesh into a UNDERWORLDS network (and optionally, creates a node) from a large range of 3D formats (including Collada, FBX, OBJ, Blender ${ }^{\sqrt{4}}$

2) Physics simulation: When perception modules provide objects localisation, the physical consistency of the locations is not typically enforced. For instance, objects that are supposed to lay on a table might be slightly above (or inset into) the table; or when dropping an item into a box, the robot can not update the location of the item anymore as it becomes occluded.

These issues can be alleviated by relying on a physics simulation to stabilise the position of objects: natural physics (including gravity) are simulated for a short amount of time (up to one second) ahead of time, and the objects' positions are updated accordingly. To this end, UNDERWORLDS pro-

${ }^{4}$ The underlying import capability is provided by the ASSIMP library. http://assimp.sourceforge.net/

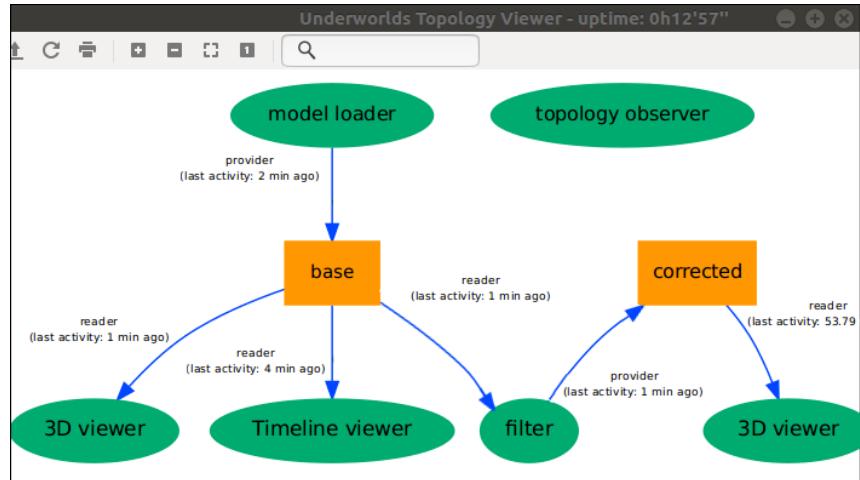

Fig. 4. Screenshot of the network topology introspection tool, with arbitrary examples of worlds (represented as boxes) and clients (ellipses) CLients are connected to the worlds either as readers or providers of data. UNDERWORLDS introspection features make it possible to also visualise when clients were last active.

vide a standard filter, the physics_filter, based on the Bullet RT physics simulation and the pybullet $5^{5}$ library. It generates an output world that mirrors its input world after a specific duration of physics simulation, the physical properties of objects (including mass, friction, inertia) being provided from standard URDF descriptions.

3) Introspection and debugging: UNDERWORLDS provides a range of tools to inspect a running network. Graphical tools (uwds explorer and uwds timeline, see Figure 47 provide a user-friendly overview of the system's graph with the connections between the clients and the worlds, as well as their activity.

Specialised command-line tools are also available to list the worlds and their content (uwds ls) at run-time, or to display detailed information for a specific node (uwds show).

4) Interface with ROS: UNDERWORLDS is meant to integrate as easily as possible into existing robot architectures, and interfaces transparently with ROS' TF frame system through the uwds $t f$ client.

The uwds tf client continuously monitors the ROS TF tree, and mirrors TF frames as nodes in the desired UNDERWORLDS world. A node is first created if none matches a given TF frame, and its transformation is subsequently updated, mirroring the TF frame. A regular expression can be provided to only mirror a subset of the TF tree into UNDERWORLDS.

Currently, the process is unidirectional: the uwds tf client performs TF to UNDERWORLDS updates, but not the reverse.

\section{Spatial Reasoning and Perspective Taking}

Spatial reasoning [20] is a field in its own right, and has been used for natural language processing for applications such as direction recognition [21], [22] or language grounding [23]. Other examples in human-robot interaction include

https://pybullet.org/ 
Ros et al. [17], [16] which has recently been integrated into a full architecture for autonomous human-robot interaction [10].

UNDERWORLDS provides an exemplary client (spatial_relations) to compute both allo-centric (independent of the viewpoint like isIn or isOn) and ego-centric (i.e., viewer-dependent, like infrontof or leftof) spatial relations between objects. Other libraries, like QSRLib [24], that implement computational models of Qualitative Spatial Relations, could be trivially combined with UNDERWORLDS to provide more advanced geometric analysis. Future developments will also include the results of the more basic research on spatio-temporal reasoning for robotics, led by de Leng and Heintz [25].

UNDERWORLDS also implements an efficient algorithm to assess object visibility from a specific viewpoint (i.e., from a given perspective node). The algorithm (color picking) enables fast (single pass) computation of the visibility of every object in the scene, while providing control regarding how many pixels should be actually visible for the object to be considered globally visible. The commandline tool uwds visibility returns the list of visible objects from the point of view of each camera in a given world, and UNDERWORLDS also provides the helper class VisibilityMonitor to programmatically access visibility information.

When integrated into a filter node, visibility computation allows easy creation of new worlds representing the estimated perspectives of the different agents.

\section{Application Example: Perspective-AWARE JOINT ACTIONS}

UNDERWORLDS is being used within the large European project MuMMER ${ }^{6}$ for service robots to compute visibility and knowledge about objects, places and agents within a mall environment.

We present here a simplified scenario, yet representative of situations which are processed in real-time by MuMMER robots: two humans and a robot are looking at a table and have to coordinate joint actions (pick and place). One object on the table (the green box in Figure 5p is only visible to one human and the robot, but hidden to the second human. The robot needs to take into account this fact to generate appropriate and legible joint manipulation actions. Figure 5 illustrates the topology of the UNDERWORLDS network that we use to this end.

A first client, static_env_provider, provides the environment models and allows to build a first ENV world where static objects, furnitures and walls are present. Then, three worlds cascade through three (independent) clients: robots_state_monitor augments ENV with the robot state (using underneath the ROS robot state publisher node) and broadcast a new world ENV_ROBOTS. objects_monitor then recognises and adds the dynamic objects (using ar_track_alvar 77, humans_monitor finally detects and

\footnotetext{
http: / / www.mummer-project.eu http://wiki.ros.org/ar_track_alvar
}
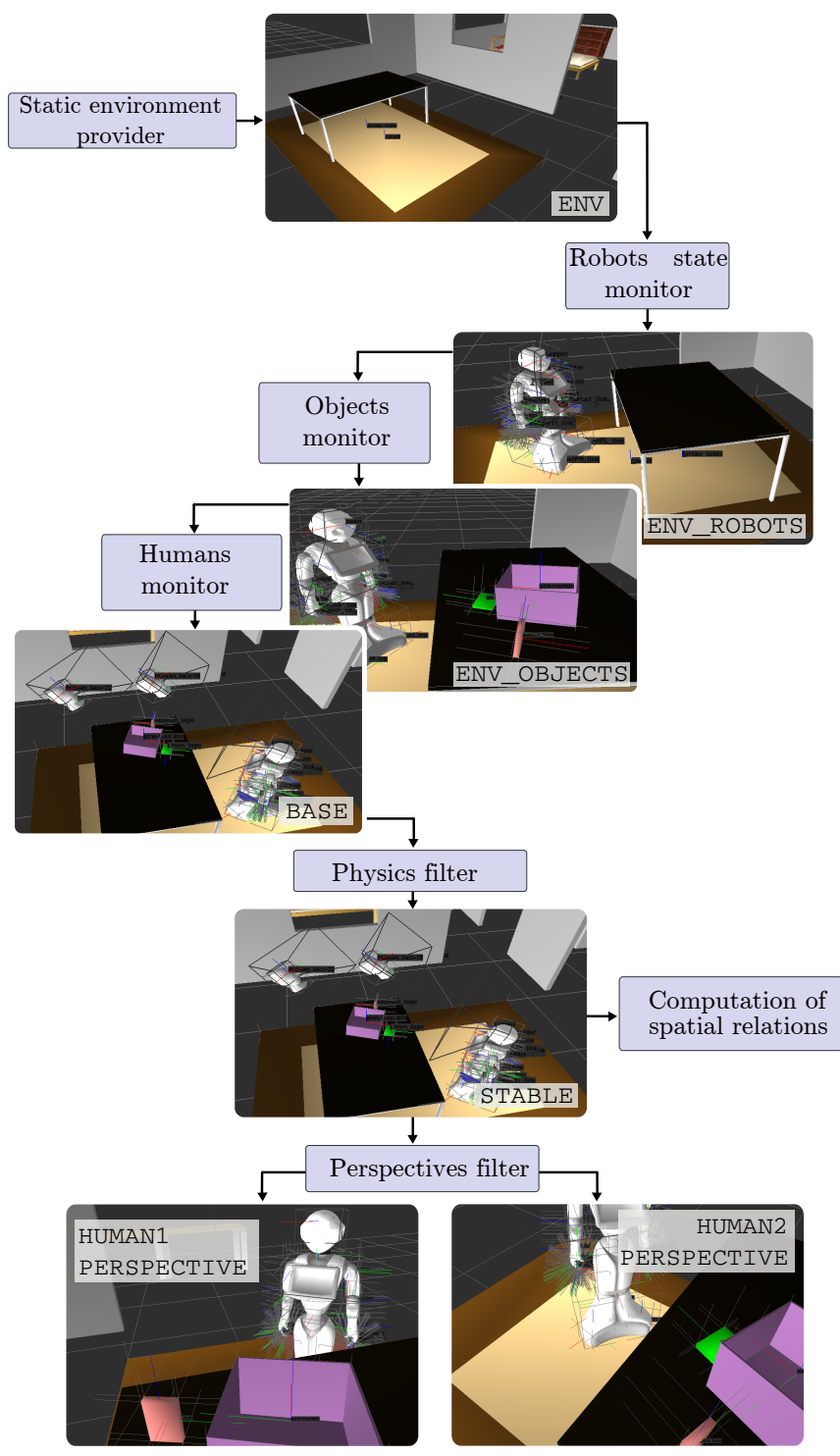

Fig. 5. Schema of the UNDERWORLDS architecture used in the MuMMER project. Clients read and generate the worlds ENV $\rightarrow$ ENV_ROBOT $\rightarrow \ldots \rightarrow$ HUMAN*_PERSPECTIVE. The last two worlds HUMAN $\{1,2\} \_P E R S P E C T I V E$ represent the immediate visual perspective of each of the humans, as well as their past visual perceptions. As such, they are the visual memories of the humans, that the robot can rely on when making decisions.

continuously updates the humans poses (using [26]). It broadcasts a world called BASE that contains as a result the static environment, the robots, the dynamic objects and the detected humans.

The world BASE goes through a physics filter client (as explained in section III-B.2 to obtain the STABLE world where all elements are present with physically-consistent locations. This physically-correct world is used by the computation_of_spatial_relations client to compute spatial relations such as onTop, is In or is Above (see Section III-C).

The world STABLE is also used by a perspectives_filter client to compute the different visual perspectives of each agent (in our case: human 1, human 2 and the robot itself). 
In addition to a $3 \mathrm{D}$ rendering of the input world from the perspective of the agent, it aggregates the history of what was visible to the agent at a given point in time. As such, it does not only offer a snapshot of the agent visual perspective at the current time but also acts as the visual memory of each agent.

With this network, the robot can easily compute that an object on the table is only seen by the human 1 and not the human 2; additionally, if human 1 moves in a position where the object is not visible anymore to him, the perspective_filter will maintain the knowledge that the human had seen it (and keep the last position where it has been seen).

UNDERWORLDS makes it possible to implement such a geometric reasoning pipeline in a fully decoupled way, and each intermediary world can be easily introspected at run-time. This example shows how UNDERWORLDS facilitates the implementation and debugging of complex spatiotemporal reasoning pipelines.

We are currently deploying a similar network in the framework of the European project MuMMER where a Pepper robot handles interactive situations in a large shopping centre in Finland. One of the situation is a guiding task where Pepper help people to find their route by pointing them landmarks and explaining them how to reach a destination. To be effective, this helping behaviour needs to be aware of the visual perspective of the human. UNDERWORLDS facilitates the implementation of such a spatio-temporal reasoning pipeline, where perception and high-level reasoning (including complex, human-aware reasoning) have to be tightly integrated. Because of the decoupling of each of the clients in the network, UNDERWORLDS also practically supports software development spread across multiple partners in different countries, with different expertise.

\section{Discussion And Conclusion}

\section{A. Relation to existing robotic middleware}

Like traditional robotic middleware, UNDERWORLDS offers a form of distributed computation based on message passing. However, it distinguishes itself from existing middlewares (including ROS extensions like DyKnow [27]) in significant ways. Most importantly, UNDERWORLDS purposefully does not offer any general capability to distribute computation and data streams amongst independent components: it focusses specifically on distributing environment models, both spatial (geometric models) and temporal (events and situations). In that sense, UNDERWORLDS really is a distributed datastructure that addresses the specific needs of spatio-temporal modelling, including the modelling of hypothetical, alternative world models, something that traditional middlewares like ROS do not address adequately. Second, and as presented above, UNDERWORLDS offers specific mechanisms for the representation and manipulation of alternative world models that are not directly achievable with traditional tools.

While using standard middleware as underlying transport for UNDERWORLDS would be technically feasible and relatively easy to implement, it does not offer any clear ad- vantage over lighter and dedicated message passing libraries like ZeroMQ or gRPC (the later being the one used by UNDERWORLDS).

\section{B. Future work}

As illustrated in section IV] UNDERWORLDS is already deployed and used on the field. Several features are however still under development.

1) Representation capabilities: as presented in section II the current version of UNDERWORLDS allows to represent objects, abstract entities like groups and perspectives. Fields are also part of the UNDERWORLDS design, but are not yet implemented. Fields are commonly used to represent continuously-valued spatial entities. Fields might or might not be spatially bounded. Examples include the working space of a robot arm (spatially bounded), the field of view of a camera (spatially bounded), proxemics (potentially unbounded). We plan to represent fields in UNDERWORLDS using the memory-efficient octomaps [28] or NDT-OM maps [29]. Similarly to geometric data,these datastructures will not be directly stored with the nodes (nodes will refer to them through handles), but unlike geometric data, they will not be treated as immutable datasets by the server, permitting real-time updates.

Representation of uncertainty: currently node positions are stored as $4 \times 4$ transformation matrices, relative to the node parent. This representation is efficient, and conveniently matches traditional representation systems (including ROS TF frames or OpenGL transformations). However, the explicit management of uncertainties is instrumental to many robotic applications, and we plan to add full support for position uncertainties to UNDERWORLDS. We plan to add this support by adding a pose covariance matrix to the nodes, and equipping the different UNDERWORLDS helper tools with corresponding support (like covariance ellipses visualisation in uwds view).

2) Implementation and Integration: we plan to continue to improve the integration of UNDERWORLDS into existing software architectures. A short-term goal is to provide excellent $\mathrm{C}++$ support, with a high-level, user-friendly $\mathrm{C}++$ client library. This is critical for a broader adoption of UNDERWORLDS within the robot community. Support for other languages might follow, depending on demands and open-source contributions.

\section{Conclusion}

We have introduced UNDERWORLDS, a novel framework for shared and composable spatio-temporal representations of a robot's world. The key contributions of our approach are: a composite data structure for environment representation within a robotic software architecture, made of a scene graph and a timeline; a mechanism to efficiently and transparently share this data structure amongst a set of clients (the software modules of the robot); a cascading architecture permitting the explicit of representation of alternative states of the world while maintaining a network of dependencies. 
We have additionally presented a concrete instantiation of a system relying on UNDERWORLDS for its representation needs, and we have sketched future directions of development.

We believe this work can practically support existing robotic architectures with state-of-the-art spatio-temporal representation capabilities. We also hope that this line of research can lead to a better understanding of the representation needs of modern robotic systems, and participate to the emergence of a possible common representation platform for robots, building on previous formalisation efforts like the RSG-DSL domain specific language [30].

\section{ACKNOWLEDGMENT}

This work has been supported by the EU H2020 Marie Skłodowska-Curie Actions project DoRoThy (grant 657227), the EU H2020 MuMMER project (grant 688147) and the EU H2020 L2TOR project (grant 688014).

\section{REFERENCES}

[1] H. Jacobsson, N. Hawes, G.-J. Kruijff, and J. Wyatt, "Crossmodal content binding in information-processing architectures," in Proceedings of the 3rd ACM/IEEE International Conference on Human Robot Interaction. New York, NY, USA: ACM, 2008, pp. 81-88.

[2] N. Mavridis and D. Roy, "Grounded situation models for robots: Where words and percepts meet," in IEEE/RSJ International Conference on Intelligent Robots and Systems, 2006.

[3] A. Nüchter and J. Hertzberg, "Towards semantic maps for mobile robots," Robotics and Autonomous Systems, vol. 56, no. 11, pp. 915 $-926,2008$.

[4] C. Galindo, J. Fernández-Madrigal, J. González, and A. Saffiotti, "Robot task planning using semantic maps," Robotics and Autonomous Systems, vol. 56, no. 11, pp. 955-966, 2008.

[5] N. Blodow, L. C. Goron, Z.-C. Marton, D. Pangercic, T. Rühr, M. Tenorth, and M. Beetz, "Autonomous semantic mapping for robots performing everyday manipulation tasks in kitchen environments," in 2011 IEEE/RSJ International Conference on Intelligent Robots and Systems (IROS), San Francisco, CA, USA, September, 25-30 2011.

[6] C. Lörken and J. Hertzberg, "Grounding planning operators by affordances," in International Conference on Cognitive Systems (CogSys), 2008, pp. 79-84.

[7] K. Varadarajan and M. Vincze, "Ontological knowledge management framework for grasping and manipulation," in IROS Workshop: Knowledge Representation for Autonomous Robots, 2011.

[8] E. A. Sisbot, R. Ros, and R. Alami, "Situation assessment for humanrobot interactive object manipulation," in 2011 RO-MAN, July 2011, pp. $15-20$.

[9] G. Milliez, M. Warnier, A. Clodic, and R. Alami, "A framework for endowing an interactive robot with reasoning capabilities about perspective-taking and belief management," in The 23rd IEEE International Symposium on Robot and Human Interactive Communication, 2014, pp. 1103-1109.

[10] S. Lemaignan, M. Warnier, E. A. Sisbot, A. Clodic, and R. Alami, "Artificial cognition for social human-robot interaction: An implementation," Artificial Intelligence, 2016.

[11] M. Beetz, M. Tenorth, and J. Winkler, "Open-EASE - a knowledge processing service for robots and robotics/ai researchers," in Robotics and Automation (ICRA), 2015 IEEE International Conference on. IEEE, 2015, pp. 1983-1990.

[12] M. Naef, E. Lamboray, O. Staadt, and M. Gross, "The blue-c distributed scene graph," in Proceedings of the workshop on Virtual environments 2003. ACM, 2003, pp. 125-133.

[13] S. Blumenthal, H. Bruyninckx, W. Nowak, and E. Prassler, "A scene graph based shared 3d world model for robotic applications," in 2013 IEEE International Conference on Robotics and Automation, May 2013, pp. 453-460.

[14] P. Bustos, L. J. Manso, J. P. Bandera, A. Romero-Garcés, L. V. Calderita, R. Marfil, and A. Bandera, "A unified internal representation of the outer world for social robotics," in Robot 2015: Second Iberian Robotics Conference. Springer, 2016, pp. 733-744.
[15] M. Beetz, D. Jain, L. Mösenlechner, and M. Tenorth, "Towards performing everyday manipulation activities," Robotics and Autonomous Systems, vol. 58, no. 9, pp. 1085-1095, 2010.

[16] R. Ros, S. Lemaignan, E. A. Sisbot, R. Alami, J. Steinwender, $\mathrm{K}$. Hamann, and F. Warneken, "Which one? grounding the referent based on efficient human-robot interaction," in 19th IEEE International Symposium in Robot and Human Interactive Communication, 2010.

[17] R. Ros, E. A. Sisbot, R. Alami, J. Steinwender, K. Hamann, and F. Warneken, "Solving ambiguities with perspective taking," in Proceedings of the 5th ACM/IEEE international conference on Humanrobot interaction. IEEE Press, 2010, pp. 181-182.

[18] S. Lemaignan and P. Dillenbourg, "Mutual modelling in robotics: Inspirations for the next steps," in Proceedings of the 2015 ACM/IEEE Human-Robot Interaction Conference, 2015.

[19] K. Von Fintel, "What is presupposition accommodation, again?" Philosophical perspectives, vol. 22, no. 1, pp. 137-170, 2008.

[20] J. O'Keefe, The Spatial Prepositions. MIT Press, 1999.

[21] T. Kollar, S. Tellex, D. Roy, and N. Roy, "Toward understanding natural language directions," in HRI, 2010, pp. 259-266.

[22] C. Matuszek, D. Fox, and K. Koscher, "Following directions using statistical machine translation," in Proceedings of the International Conference on Human-Robot Interaction. ACM Press, 2010.

[23] S. Tellex, "Natural language and spatial reasoning," Ph.D. dissertation, MIT, 2010.

[24] Y. Gatsoulis, M. Alomari, C. Burbridge, C. Dondrup, P. Duckworth, P. Lightbody, M. Hanheide, N. Hawes, D. Hogg, A. Cohn et al., "Qsrlib: a software library for online acquisition of qualitative spatial relations from video," Tech. Rep., 2016.

[25] D. de Leng and F. Heintz, "Qualitative spatio-temporal stream reasoning with unobservable intertemporal spatial relations using landmarks." in $A A A I, 2016$, pp. 957-963.

[26] V. Khalidov and J.-M. Odobez, "Real-time multiple head tracking using texture and colour cues," Idiap, Idiap-RR Idiap-RR-02-2017, 22017.

[27] D. de Leng and F. Heintz, "DyKnow: A Dynamically Reconfigurable Stream Reasoning Framework as an Extension to the Robot Operating System," in IEEE Simulation, Modeling, and Programming for Autonomous Robots (SIMPAR), 2016, pp. 55-60. [Online]. Available: http://urn.kb.se/resolve?urn=urn:nbn:se:liu:diva-132266

[28] A. Hornung, K. M. Wurm, M. Bennewitz, C. Stachniss, and W. Burgard, "Octomap: An efficient probabilistic 3d mapping framework based on octrees," Autonomous Robots, vol. 34, no. 3, pp. 189-206, 2013.

[29] J. P. Saarinen, H. Andreasson, T. Stoyanov, and A. J. Lilienthal, "3d normal distributions transform occupancy maps: An efficient representation for mapping in dynamic environments," The International Journal of Robotics Research, vol. 32, no. 14, pp. 1627-1644, 2013.

[30] S. Blumenthal and H. Bruyninckx, "Towards a domain specific language for a scene graph based robotic world model," arXiv preprint arXiv:1408.0200, 2014. 